\title{
Caracterización y asociación del rendimiento físico en futbolistas chilenos con parálisis cerebral \\ Characterization and association of the physical performance of Chilean football players with cerebral palsy
}

${ }^{1} \mathrm{M}$ atías Henríquez, ${ }^{2}$ Felipe Herrera, ${ }^{3}$ Fernando M uñoz, ${ }^{4} \mathrm{C}$ ristian Luarte Rocha, ${ }^{5}$ M ónica Fernández, ${ }^{6} \mathrm{D}$ aniel Bueno, ${ }^{7}$ Carolina Zapata Huenullan, 8 oão Paulo Borin, ${ }^{9}$ Luis Felipe Castelli Correia de Campos

${ }^{1}$ Instituto nacional de rehabilitación Pedro Aguirre Cerda (Chile) ${ }^{2}$ UniversidadViña del M ar (Chile), ${ }^{3} U$ niversidad Metropolitana de Ciencias de la Educación (Chile), ${ }^{4} U$ niversidad San Sebastián (Chile), 5U niversidad Adventista de Chile (Chile), ${ }^{6}$ Centro deAlto Rendimiento-CAR (Chile), ${ }^{7} U$ niversidad Santo Tomás (Chile), ${ }^{8}$ U niversidad Estatal de Campinas (Brasil), 9 Universidad del Bío-Bío (Chile)

Resumen. 0 bjetivo: el objetivo de este estudio fue: i) Caracterizar el perfil del rendimiento físico de los futbolistas con parálisis cerebral (PC) de la selección chilena de fútbol 7 acorde a su clasificación deportiva (CD) y posición de juego; ii) Analizar la relación entre las variables de rendimiento físico con la CD. Método: Participaron 19 jugadores varones con PC pertenecientes a la selección chilena de fútbol 7 agrupados según CD (FT1, FT2, FT3) y posición de juego. Se realizaron mediciones de agilidad (AG), capacidad de realizar sprint repetido (RSA), distancia recorrida en la pruebaYo-Yo (D), capacidad de salto con las pruebas Squat J ump (SI) y Countermovement Jump (CMJ). Resultados: Se encontraron diferencias significativas de acuerdo a las CD donde los jugadores FT 3 lograron un mayor rendimiento en las pruebas de AG y D comparado con FT2 y FT1, no así para RSA donde solo fue superior a FT1. Mediocampistas lograr on un mayor rendimiento con un tamaño del efecto grande en todas las variables en comparación a las posiciones de defensa y delantero. Se observaron relaciones fuertes y muy fuertes entre todas las variables de rendimiento físico, así también para las relaciones con la CD a excepción de SJ y CMJ. Conclusión: Se deben considerar las características específicas de cada CD de los jugadores con PC en conjunto con la posición de juego y la táctica del equipo en sí misma, conjugando una serie de factores que busquen lograr los más al tos niveles de rendimiento físico y deportivo.

Palabras Clave: Condición física, Lesión cerebral, Paralímpico, Capacidad Anaeróbica, Capacidad Aeróbica.

\begin{abstract}
The aims of this study were: i) Characterize the physical performance profile of cerebral palsy (CP) football players of the Chilean national team of football 7 a-Side according to the sport classification (SC) and playing position; ii) Analyze the relationship between physical performance variables and the SC. M ethod: 19 male football players with CP belonging to the Chilean national team of football 7 a-Side participated and were grouped according to SC (FT2; FT2; FT3) and playing position. Measurements of A gility (AG), repeated sprint ability (RSA), the covered distance in the Yo-Yo test (D), jump ability in Squat Jump (S)), and Countermovement Jump (CMJ) test were made. Results: Significant differences were found according to SC, where FT3 players achieved higher performance in AG and D tests compared to FT2 and FT1, not for RSA, which was only higher than FT1. Midfielder achieved a higher performance with a large effect size in all variables compared with defender and forward positions. Strong and very strong relationships were observed between all the physical performance variables, as well as for relationships with SC except for SI and CMJ. Conclusion: The specific characteristics of each SC of CP players must be considered in conjunction with the playing position and the team's tactics, combining a series of factors that seek to achieve the highest levels of physical and sports performance.
\end{abstract} Keywords: Physical Condition, Brain injury, Paralympic, Anaerobic capacity, A erobic capacity.

\section{Introducción}

La participación de personas con discapacidad en el fútbol ha crecido en los últimos años a nivel mundial, destacándose un gran interés en el campo de las ciencias del deporte por favorecer el rendimiento deportivo

Fecha recepción: 15-06-20. Fecha de aceptación: 15-10-20 Luis Felipe Castelli Correia de Campos

Icastelli@ ubiobio.cl
(A hmed, Hussain, Beasley, Dvorak, \& Weiler, 2015). Dentro de las distintas modalidades de fútbol para personas con discapacidad, el fútbol 7 se destacapor ser uno de los escasos deportes colectivos para personas con parálisis cerebral (PC), el cual es similar al fútbol convencional (para personas sin discapacidad) pero que se rige bajo una serie de adaptaciones relacionadas a modificaciones en el campo de juego, tiempo de juego, dimensiones del arco, saque lateral y regla de fuera de juego (IFCPF, 2018a; W illick et al., 2013). Las adapta 
ciones realizadas para la ejecución del juego están rela cionadas principalmente a la adecuación según el tipo de discapacidad que presentan los deportistas, el cual es caracterizado por presentar un compromiso neurológico con consecuencias en el desempeño motor (Boyd et al., 2016). El fútbol 7 se rige de acuerdo a los lineamientos del comité paralímpico internacional el cual define que la presencia de ciertos déficits permite que los deportistas sean seleccionados para la práctica competitiva (Tweedy \&Vanlandewijck, 2011). Ligado a esto, deportistas con condiciones de salud afectados por hipertonía, ataxia y/ 0 atetosis pueden llegar a ser el egibles para la participación en la disciplina fútbol 7, cumpliendo previamente criterios específicos del deporte todo esto en el contexto de una regulación estructural a través de la clasificación deportiva (CD) (IFCPF, 2018b). Actualmente este deporte categoriza a sus participantes identificando el impacto de la deficiencia en la limitación de la actividad, proponiendo 3 clases deportivas (FT1, FT2 y FT3) diferenciadas según el grado decompromiso motor y adecuando las condiciones específicas de competencia (IFCPF, 2018b). Durante el paso de los años, distintos deportes paralímpicos han ganado una gran popularidad y desarrollo del cual también se ha hecho parte el fútbol 7, sin embargo existe una limitada informa ción acerca de la descripción de las características antropométricas y el desempeño motor de estos mismos deportistas (Reina, Iturricastillo, Castillo, U rbán, \&Yanci, 2020).

Distintos estudios han analizado el desempeño de jugadores con PC, describiendo diferencias en su rendimiento de acuerdo a su CD y perfil funcional. Se desta ca que los jugadores que presentan mayores déficits motores exhiben un peor rendimiento en comparación a jugadores con menores compromisos durante la realización de pruebas de rendimiento físico (Pastor, Campayo-Piernas, Pastor, \& Reina, 2019; Yanci, CastiIlo, Iturricastillo, \& Reina, 2019). Generalmente estas diferencias están en el contexto de las deficiencias presentes en personas con PC como la disminución del volumen muscular, co-contracción, espasticidad, contracturas y cambios metabólicos que afectan tareas específicas al deporte (Barrett \& Lichtwark, 2010; Graham et al., 2016; Verschuren et al., 2018).

El fútbol es una disciplina compleja debido a la naturaleza del deporte mismo, esto debido a los múltiples requerimientos físicos y a la contribución de diferentes sistemas energéticos que permite realizar actividades motoras complejas (M organs, O rme, Anderson, \& Drust, 2014). El fútbol 7 al igual que el fútbol conven- cional demanda actividades de alta intensidad, donde en partidos internacionales se ha descrito que los jugadoreshan al canzado una distancia total de 4342.7 \pm 1808.4 $m$ (Yanci et al., 2019), carreras o sprint de alta velocidad $\left(>18 \mathrm{~km} \mathrm{~h}^{-1}\right)$ con una cobertura de distancia recorrida de $3.27 \pm 1.96$ m (Yanci, Castillo, Iturricastillo, Urbán, \& Reina, 2018) y velocidades máximas promedio de hasta $22.95 \pm 2.01 \mathrm{~km} \mathrm{~h}^{-1}$ (Reina et al., 2020).

El control y monitorización del entrenamiento resulta ser una actividad fundamental para lograr niveles óptimos de rendimiento, analizando las características intermitentes del fútbol, la necesidad de requerimientos aeróbicos/ anaeróbicos y el contexto del compromiso motor que presentan los jugadores con PC (Gorla et al., 2019; M organs et al., 2014). La realización de pruebas que permitan evaluar la capacidad aeróbica como el test Yo-Yo ha sido ampliamente difundida en el mundo del fútbol, siendo también utilizada en futbolistas con PC, donde su rendimiento ha demostrado ser bajo en comparación con futbolistassin discapacidad, presentando diferencias de acuerdo a la CD y posición de juego (Kloyiam, Breen, Jakeman, Conway, \& Hutzler, 2011). Por otro lado, actividades asociadas a la potencia anaeróbica láctica y aláctica mediante la realización de carreras de alta velocidad de forma rectilínea o con cambios de dirección, aceleración/ desaceleración y saltos, resultan ser principales características y acciones determinantes durante el juego (Stølen, Chamari, Castagna, \& W isløff, 2005).

Estudios previos han descrito que de acuerdo a la $C D$ de cadajugador existen diferencias en el rendimiento asociado a la capacidad de salto (Reina, Iturricastillo, Sabido, Campayo-Piernas, \&Yanci, 2018), capacidad de realizar cambios de dirección (Reina, Sarabia, Yanci, García-Vaquero, \& Campayo-Piernas, 2016), capacidad de aceleración/ desaceleración y carreras de alta velocidad (Reina, Sarabia, Caballero, \&Yanci, 2017), a pesar de ello, no se han observado estudios que identifiquen las variables mencionadas en el contexto de la posición de juego en futbolistas con PC. En base a esto, el objetivo de este estudio fue: i) Caracterizar el perfil del rendimiento físico de los futbolistas con PC de la selección chilena de fútbol 7 acorde a su CD y posición de juego; ii) Analizar la relación entre las variables de rendimiento físico con la CD.

\section{Metodología}

\section{Participantes}

En este estudio participaron 19 jugadores de sexo 
masculino pertenecientes a la selección chilena de fútbol 7 que estuvieron en un periodo de entrenamiento en preparación a una competencia internacional (edad=25.3 \pm 7.1 años; peso corporal $=68.1 \pm 9.3 \mathrm{~kg}$; talla $=170 \pm 0.1 \mathrm{~cm})$. Para el estudio se consideró sola mente jugadores de campo, siendo agrupados de acuerdo ala posición que ocupaban en defensas (DEF), medio campistas (MC) y delanteros (DEL). Todos los atletas presentaron CD (FT1, FT2, FT3) de acuerdo a la normativa declarada por la federación internacional de fútbol parálisis cerebral (IFCPF, 2018b). La cantidad de atletas para cada grupo de CD, topografía del compromiso motor y posición de juego son presentadas en la tabla 1.

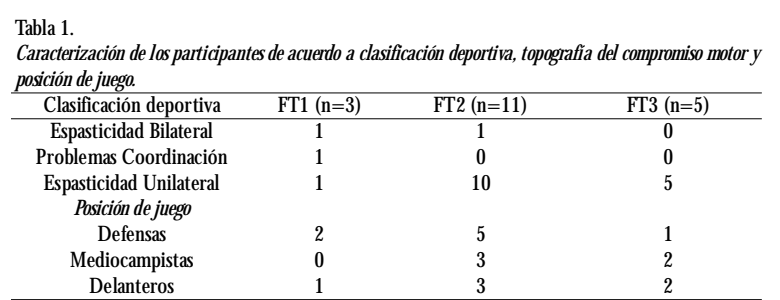

Los participantes fueron informados acerca de los objetivos del estudio, las implicancias de su participa ción y se explico acerca de la posibilidad de retirarse en cualquier momento de la evaluación sin contemplación de ningún tipo de multa. Este estudio fue aprobado por el comité de ética de la universidad Santo Tomás y estuvo concordancia con los principios de la declaración de Helsinki.

\section{Procedimientos}

Todos los jugadores fueron familiarizados con los procedimientos desarrollados en este estudio, los cuales forman parte del programa de evaluación de la condición física de la selección chilena de fútbol 7. Las mediciones se realizaron en tres días diferentes, en hora rios similares y separados por un día de diferencia con el fin de no ver afectado el rendimiento físico por la presencia de fatiga. Para esto, en el día 1 se realizaron las pruebas de salto vertical y agilidad, en el día 2 se realizó la prueba de potencia anæeróbica y en el día 3 la prueba de capacidad y potencia aeróbica. Todas las pruebas se efectuaron en cancha de pasto sintético, excepto los saltos que se realizaron en una superficie estable. Se instruyó a los jugadores a no ejecutar ningún tipo de actividad física moderada o vigorosa el día previo a la evaluación y a realizar las pruebas a una máxima intensidad. Previo a las mediciones, todas las actividades comenzaron con un calentamiento estandarizado de 15 minutos consistiendo en ejercicios de movilidad articu- lar, carreras y zancadas de baja intensidad, cambios de dirección y carreras de aceleración/ desaceleración auna intensidad incremental (Mujika, Santisteban, Impellizzeri, \& Castagna, 2009).

\section{Agilidad}

Para estimar la agilidad (AG) se utilizó la prueba Illinois la cual presenta una buena confiabilidad (CCl= 0.96) en futbolistas con PC (Reina et al., 2016). El circuito determinado consistió en que el atleta realiza una carrera de 10 m, gira en dirección a la línea de inicio, donde luego de retornar a esta misma, el participante ejecuta un slalom entre cuatro conos, completando dos carreras de 10 metros para finalizar la prueba (Reina et al. , 2016). Los tiempos se cal cularon con dos fotoceldas (PEM 10D, Velleman, Gavere, Bélgica) ubicadas en la línea de inicio y final en conjunto con un sistema de interface (Chronopic, Chronojump Boscosystem, Barcelona, España). Se realizaron tres repeticiones de la prueba con 3 minutos de descanso entre cada intento, para el análisis solo se consideró el mejor intento medido en segundos ( $s$ ).

\section{Potencia Anaeróbica}

La potencia anaeróbica fue evaluada por medio de la capacidad de realizar sprint repetido (RSA) mediante el protocolo descrito por Buchheit, M endez-Villanueva, Delhomel, Brughelli \& Ahmaidi (2010). La prueba consiste en realizar 6 repeticiones de sprint a máxima intensidad en una distancia de $30 \mathrm{~m}$ (15 m de sprint + giro de $180^{\circ}+15 \mathrm{~m}$ sprint). Cada circuito de carrera se encuentra separado por 20 segundos de recuperación pasiva, con los deportistas en posición de pie entre cada repetición. Lostiempos se calcularon con dosfotoceldas (PEM 10D, Velleman, Gavere, Bélgica) ubicadas en la única línea de inicio y final en conjunto con un sistema de interface (Chronopic, Chronojump Boscosystem, Barcelona, España). Se calculó el mejor sprint $\left(R_{S A}\right)$ y el promedio de los sprint (RSA $)_{p}$.

\section{Capacidad Aeróbica}

La capacidad aeróbica se estimó a través del protocolo Yo-Yo intermitente nivel 1, prueba ampliamente utilizada en futbolistas con y sin discapacidad (K loyiam et al. , 2011; Köklü, Asçi Koçak,Alemdarođlu, \& Dündar, 2011). La prueba consiste en realizar carreras de ida y vuelta sobre un tramo de $20 \mathrm{~m}$, a una velocidad que aumenta de forma progresiva determinada por una señal auditiva, presentando periodos de recuperación activa entre las carreras hasta llegar a la exhaustación. La 
prueba fue considerada final izada cuando el participante falla 3 veces consecutivas en alcanzar la línea de frente en el tiempo adecuado o presentar fatiga excesiva. Se consideró la distancia recorrida (D) total al canzada por cada participante (Castagna, Impellizzeri, Chamari, Carlomagno, \& Rampinini, 2006).

\section{SaltosVerticales}

Las variables de desempeño neuromuscular fueron evaluadas por medio del salto vertical mediante las pruebas Squat Jump (S) ) y Countermovement Jump (CMJ) sin asistencia de los brazos, a través de la plataforma de contacto (DMJU M P $2.5^{\circledR}$ ) con sistema bluethooth para smartphone. Se consideró el protocolo establecido por Bosco, Luhtanen, \& Komi (1983), para la ejecución de ambas pruebas. Se realizaron 3 intentos con intervalos de un minuto de descanso, registrándose el mayor valor obtenido respecto a la altura al canzada. Para aquellos deportistas que presentaban espasticidad en un hemicuerpo y dificultad para fijar las manos a las caderas, se les permitió mantener las manos a un costado de su cuerpo sin inferir esto en los resultados (Yanci et al., 2016).

\section{Análisis estadístico}

Para el análisis de los datos se utilizó el GraphPad Prism ${ }^{\circledR} 8.2$ (San Diego, CA, USA) y Excel ${ }^{\circledR} 2019$. Los resultados se presentaron por medio del promedio y la desviación estándar (DE). La normalidad de los datos se determinó mediante la prueba de Shapiro-W ilk y la homogeneidad por medio de la prueba de Levene. Se realizó un análisis de varianza unifactorial (one-way ANOVA) con un post hoc deTukey para examinar las diferencias medias entre los grupos según $C D$ y según posición de juego. Se utilizó la prueba t para muestras independientes para la comparación entre pares. Las relaciones entre las variables del rendimiento físico y la $C D$, se evaluaron utilizando la correlación lineal de Pearson y Spearman, respectivamente (r). Se utilizó la siguiente escala de magnitudes para evaluar los coeficientes de correlación: <.09, trivial; . 10 -.29, pequeño; $<.30-.49$, moderado; <.50-.69, fuerte; <.70-.89, muy fuerte y $<.90-1.0$, casi perfecto. Se calculó el tamaño de efecto (TE) para la comparación entre pares (Cohen, 1988) y se consideró los valores sugeridos por Rhea: por encima de 1.0, entre 1.0 y .50, entre .50 y .25 y por debajo de .25 se consideraron grandes, moderados, pequeño y trivial, respectivamente (Rhea, 2004). El nivel de significancia considerado fue determinado como $p<$ 0.05 .

\section{Resultados}

En la tabla 2 y 3 son presentados los resultados de las pruebas de rendimiento físico según la $C D$ y posición de juego. Se verificó que el grupo FT 3 fueron más ágiles y recorrieron una mayor distancia en la prueba de resistencia intermitente comparado a los jugadores FT 1 (AG: $p=.00, T E=2.9$, [grande], $D: p=.00, T E=1.6$, [grande]) y los jugadores FT2 (AG: $p=.02, T E=1.5$, [grande], $\mathrm{D}: \mathrm{p}=.00, \mathrm{TE}=1.2$, [grande]). FT3 también presentaron una mejor capacidad de realizar sprint con cambio de dirección (RSA $: p=.04, T E=2.2$, [grande]; $R S A_{p}: p=.04, T E=2.0$, [grande]), al compararlo con los jugadores FT1. No se observaron diferencias significa tivas entre las variables de saltos verticales SJ y CMJ, según la CD.

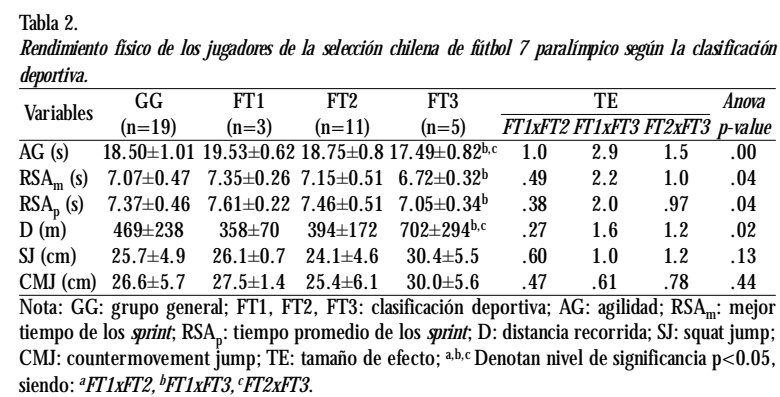

Asociado a la posición de juego, se observó que los jugadores $M C$ recorrieron la mayor distancia en la prueba de resistencia intermitente comparado a los DEF ( $D: p=.00, T E=2.6$, [grande]) yDEL ( $D: p=.03, T E=1.5$, [grande]) y también, fueron significativamente más ágiles (AG: $p=.01, T E=1.8$, [grande]). J ugadores MC también presentaron mejores resultados en la capacidad de real izar sprint repetido con cambio de dirección $\left(\mathrm{RSA}_{\mathrm{m}}\right.$ : $p=.00, T E=2.1$, [grande]; $R S A_{p}: p=.02, T E=1.8$, [grande]), y mayor altura de salto en contra movimiento (CM): $p=.01, T E=2.8$ [grande]), comparado a los DEF.

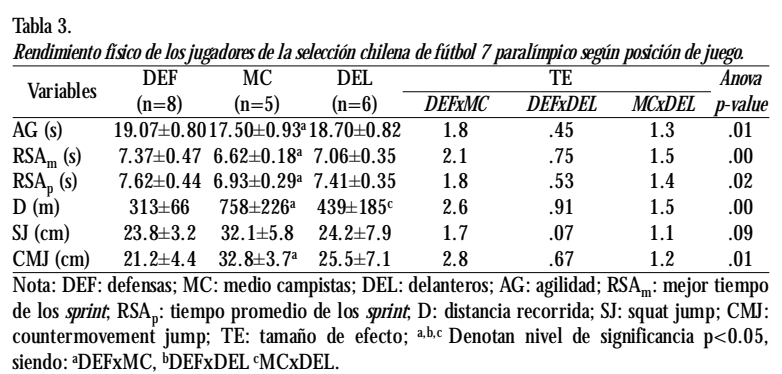

En la tabla 4 son presentados los resultados de la relación entre variables de rendimiento físico en los atletas y en la figura 1 las relaciones de las variables con la CD. Se observaron relaciones fuertes y muy fuertes entre todas las variables de rendimiento físico. La AG 
presentó relaciones significativas positivas con las varia bles de RSA ( $r=>.81$ y <.84) e inversamente proporcionales con la distancia recor rida y los saltos verticales $(r=>-.51$ y $<-.75)$. Junto con esto, las variables de $R S A_{m}$ y $R S A_{p}$ presentaron relaciones significativas e inversamente proporcionales con el resto de las varia bles del estudio $(r=>-.55$ y $>-.91)$.

Tabla 4.

Correlación de Pearson entre las variables de rendimiento físico de los de la selección chilena de fútbol 7 paralímpico.

\begin{tabular}{|c|c|c|c|c|c|c|}
\hline & & $\mathrm{RSA}_{\mathrm{m}}$ & $\mathrm{RSA}_{p}$ & D & SJ & CMJ \\
\hline AG & $r$ & .84 & .81 & $\begin{array}{l}-.75 \\
\end{array}$ & $\begin{array}{l}-.56 \\
\end{array}$ & -.51 \\
\hline & $p$-value & .000 & .000 & .000 & .030 & .044 \\
\hline $\mathrm{RSA}_{\mathrm{m}}$ & $\begin{array}{c}r \\
p \text {-value }\end{array}$ & & $\begin{array}{l}.97 \\
.000\end{array}$ & $\begin{array}{l}-.91 \\
.000\end{array}$ & $\begin{array}{l}-.63 \\
.014\end{array}$ & $\begin{array}{l}-.63 \\
.014\end{array}$ \\
\hline $\mathrm{RSA}_{\mathrm{p}}$ & $\begin{array}{c}r \\
p \text {-value }\end{array}$ & & & $\begin{array}{l}. .89 \\
.000\end{array}$ & $\begin{array}{c}-.55 \\
0,035\end{array}$ & $\begin{array}{l}-.58 \\
.025\end{array}$ \\
\hline D & $\begin{array}{c}r \\
p \text {-value }\end{array}$ & & & & $\begin{array}{l}.76 \\
.002 \\
\end{array}$ & $\begin{array}{l}.75 \\
.002 \\
\end{array}$ \\
\hline SJ & $\begin{array}{c}r \\
p \text {-value }\end{array}$ & & & & & $\begin{array}{l}.89 \\
.000\end{array}$ \\
\hline
\end{tabular}

En relación a la $C D$, se observó una relación significativa e inversamente proporcional con las variables de AG $(r=-.74), R_{m}(r=-.50)$ y $\operatorname{RSA}_{p}(r=-.59)$, sugiriendo que jugadores con un mayor compromiso son menos ágiles, más lentos y presentan una menor capa cidad para realizar sprint con cambios de dirección. Por otro lado, se observó que la mayor distancia recorrida fue realizada por atletas con un menor grado de déficit motor ya que la $\mathrm{D}$ presentó correlaciones significativas y positivascon laCD $(r=.51)$. Finalmenteno se observaron relaciones significativas para las variables de saltos verticales (S) y CMJ) con CD.
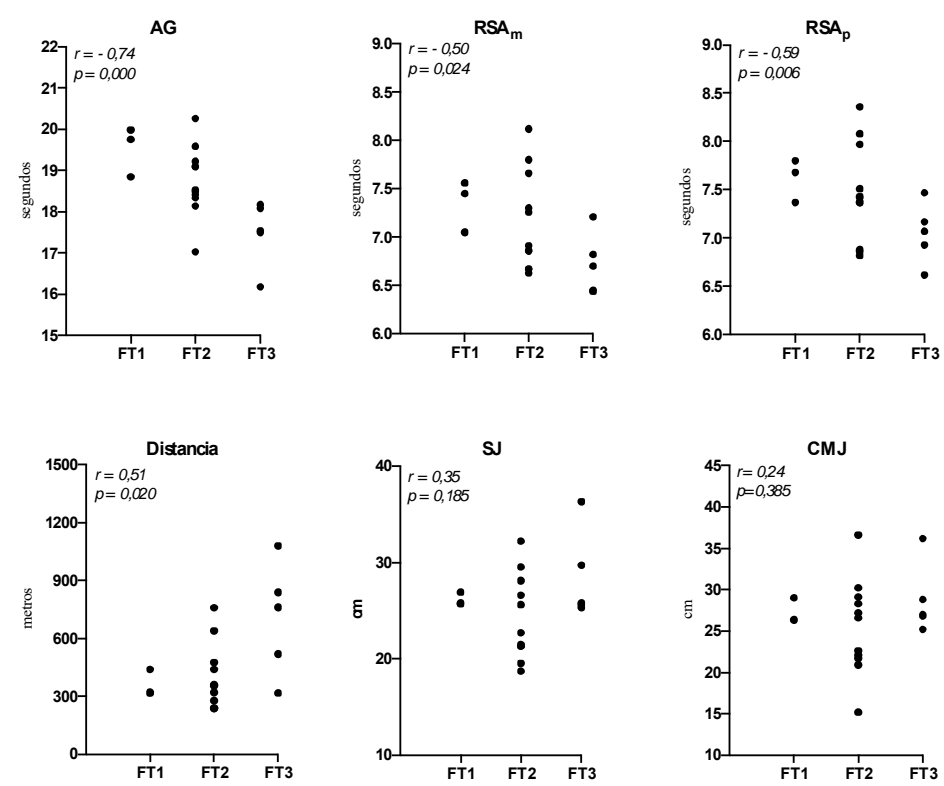

Figura 1. Correlación de Spearman entre las variables del rendimiento físico y la clasificación deportiva de los jugadores de la selección chilena de fútbol 7 paralímpico.

Nota: AG: agilidad; $\mathrm{RSA}_{\mathrm{m}}$ : mejor tiempo de los sprint; RSA $\mathrm{p}_{\mathrm{p}}$ : tiempo promedio de los sprint; D: distancia recorrida; SJ : squat jump; CMJ: countermovement jump.

\section{Discusión}

Los objetivos de este estudio fueron caracterizar el perfil de rendimiento físico de futbolistas con PC de la selección chilena de fútbol 7 paralímpico, según CD y posición de juego. El segundo objetivo fue analizar la relación entre las variables de rendimiento físico con la CD. Los principales resultados obtenidos evidenciaron que los atletas FT3 lograron mejores resultados en la prueba de $A G, R S A\left(R_{m} A_{m}\right.$ y $\left.R S A_{p}\right)$ y distancia recorrida en la prueba de capacidad aeróbica. En relación a la posición de juego, los MC demostraron un mejor rendimiento físico para las variables de agilidad, capacidad de realizar sprint repetidos, capacidad de recorrer una mayor distancia en la prueba de capacidad aeróbica y unamayor alturadel salto con contramovimiento (CMJ).

La velocidad y la agilidad son capacidades complejas que involucran movimientos rápidos del cuerpo pero que están diferenciadas por la incorporación de cambios de dirección, lo cual es una condicionante que se ha descrito importante en el fútbol (Chaouachi et al., 2012; Horicka, Hianik, \& Šimonek, 2014), siendo esto también parte de las acciones determinantes durante un partido de fútbol para deportistas con PC (Yanci et al., 2019, 2018). Para el análisis entre CD, se pueden observar diferencias donde principalmente los jugadores FT3 presentan un mayor rendimiento en comparación con los jugadores FT 2 y FT1. Estos resultados están en concordancia con lo descrito por otros autores donde los jugadores de las clases que presentan un mayor compromiso motor pueden presentar mayores limitaciones en la ejecución de tareas relacionadas a procesos de aceleración, desaceleración y rápidos cambios de dirección, presentando aś los resultados más bajos (Reinaet al., 2016;Yanci et al. , 2018). Estas diferencias podrían deberse a que los jugadorescon PC presentan un mayor compromiso motor, viéndose más afectados por la presencia de debilidad muscular, cocontracción y espasticidad, alterando principalmente la respuesta ante la solicitud de la generación de fuerza muscular excéntrica por parte del sistema neuromuscular en este tipo de acciones (Hussain, 0 nambele, W illiams, \& M orse, 2014; Reina et al. , 2017). Por otra parte y de acuerdo a la posición de juego, se puede observar que los jugadores de la posición MC obtuvieron un mejor rendimien- 
to en la habilidad para realizar cambios de dirección en comparación alosDEL y losDEF. Corroborando así con lo mencionado anteriormente, donde pareciera que los jugadores MC en esta disciplina presentan una mayor demanda de la agilidad para realizar cambios de dirección a alta velocidad con el fin de esquivar rivales, distribuir el juego y ejecutar acciones ofensivas, características relevantes las cuales pueden diferenciar a esta posición (Gamonales, León, Jiménez, \& M uñoz-J iménez, 2019).

Durante partidos de fútbol es común que los atletas realicen acciones de alta intensidad y en ocasiones presenten una recuperación parcial entre estímulos. En niveles competitivos se observa que los atletas deben presentar una buena capacidad para realizar sprint repetidos con cambios de dirección, siendo esto considerado como un aspecto relevante para tener una ventaja competitiva (Bishop, Girard, \& M endez-Villanueva, 2011). El rendimiento en la capacidad de realizar RSA involucra importantes aportes desde los sistemas energéticos del sistema oxidativo y componentes del sistema anaeróbico (Girard, M endez-Villanueva, \& Bishop, 2011), estas diferencias pueden responder a las consecuencias producidas por la lesión cerebral asociadas a un alargamiento de los sarcómeros musculares, cambio en el fenotipo muscular, hipertrofia de la matriz extracelular y disminución del área de sección transversal (Graham et al., 2016; Lieber \& Fridén, 2019). Yanci et al. (2018) identificaron diferencias entre las $C D$ en atletas de fútbol 7 , donde los jugadores con una mínima deficiencia logra ron realizar una distancia mayor a altas velocidades, en comparación a jugadores que presentan un mayor compromiso motor. En nuestro estudio, se observó que los atletas FT3 presentaron un mejor rendimiento en la prueba de RSA en comparación a jugadores FT1, estas diferencias están en concordancia con lo descrito en el perfil FT3, donde la potencia muscular y la velocidad de acciones con y sin balón están afectadas mínimamente (IFCPF, 2018b). Por otro lado, la prueba de RSA fue capaz de diferenciar entre posiciones de juego donde los MC presentaron un mejor rendimiento en compa ración a DEF y DEL.

En la distancia total recorrida por los atletas, se observó que esta fue menor que lo descrito en jugadores de fútbol Irlandeses con PC (K loyiam et al., 2011). Esta pruebademandaimportantessolicitudes del componente aeróbico/ anzeróbico mediante la realización de actividades de tipo intermitente, discriminando entre posición de juego, sexo y nivel de rendimiento físico (Bangsbo, laia, \& Krustrup, 2008). En el rendimiento por clasificación, los atletas FT1 y FT2 describieron menores valores en comparación a atletas FT3. Estos resultados son contrarios a lo descrito por Kloyiam et al. (2011), donde se identificó que el único jugador de la clase más «baja» (alto compromiso motor), recorrió unamayor distancia en la realización de esta misma prueba en comparación a las demás clases deportivas (Kloyiam et al., 2011). Sin embargo, existen otros estudios que han analizado la carga externa durante partidos de fútbol, donde han descrito que los jugadores que presentan clases más «altas» o que presentan una deficiencia mínima, logran cubrir una mayor distancia total (Boyd et al., 2016) y mayores distancias a una alta intensidad pudiendo describir diferencias en la potencia aeróbica acorde a cada CD (Yanci et al., 2019). Las diferencias de rendimiento en jugadores FT 1 podrían asociarse al perfil caracterizado por una mayor severidad del compromiso motor con presencia de limitaciones en el rango articular, mayor impacto de la espasticidad en la musculatura afectada, asimetrías y en al gunos casos problemas de coordinación/ planeamiento del movimiento que los diferencia del resto de las CD (IFCPF, 2018b). En personas con PC se postula que ciertos mecanismos potenciales pueden influenciar el rendimiento de la capacidad aeróbica, los cuales están relacionados con el impacto en el aumento del tono muscular, alteración en el fenotipo muscular, reclutamiento de unidades motoras y remoción del lactato durante ejercicio, pudiendo facilitar la aparición de fatiga muscular periférica temprana (Kloyiam et al., 2011; Runciman, Tucker, Ferreira, Albertus-Kajee, \& Derman, 2016). Los resultados de acuerdo al análisis de la posición de juego son consistentes con otros estudios que describen altos valores de rendimiento en la evaluación de la ca pacidad aeróbica en futbolistas de la posición MC con PC y sin PC (Kloyiam et al. , 2011; M ohr, Krustrup, \& Bangsbo, 2003; Sporis, Jukic, O stojic, \& M ilanovic, 2009). Al igual que en el fútbol convencional, pareciera ser que los deportistas que juegan en la posición MC deben responder a demandas de la posición donde requieren cubrir mayores distancias en comparación a los jugadores de las otras posiciones (Bangsbo, 2014; M ohr et al., 2003). La gran distribución de los resultados en esta prueba puede sugerir también la influencia de los factores asociados al nivel de entrenamiento, el compromiso de ladiscapacidad y diferencias según posición de juego (Moreira et al., 2021).

Para el rendimiento en la prueba de salto vertical los atletas FT 3 obtuvieron mayores valores en la al tura del salto CMJ en comparación a los jugadores FT2 y 
FT1. Los atletas FT2 no se diferenciaron de los FT1, contrario a la hipótesis de los autores ya que los atletas FT 1 deberían presentar una menor capacidad de salto y mayor compromiso motor durante su ejecución (IFCPF, 2018b; Reinaet al., 2018). Estos resultados describen la gran variabilidad en el rendimiento físico que existe entre jugadores con distintas CD y compromisos motores para esta prueba. El salto vertical puede ser efectivo en la utilización para el monitoreo de la progresión del entrenamiento y discriminación entre $C D$ en conjunto con pruebas de sal to horizontal, las cuales presentan una mejor validez por sobre la prueba ya mencionada (Reina et al. , 2018). Los resultados correspondientes a la altura de los saltos verticales (SJ y CMJ) fueron similares a los observados en seleccionados nacionales Españoles de la modalidad (Yanci et al. , 2016). En cuanto a la posición de juego, los jugadores MC obtuvieron mayores valores en la altura de salto vertical $C M$ J en comparación a los DF. La capacidad de salto vertical ha sido utilizada como una herramienta para la evaluación de la potencia muscular demostrando también una rela ción importante con el rendimiento de sprint (Loturco et al. , 2015; W isløff, Castagna, Hel gerud, Jones, \& H off, 2004). Estas características pueden justificar los resul ta dos del presente estudio, ya que los MC fueron los jugadores que presentaron un mejor rendimiento en las pruebas de acciones de alta intensidad comparado a los jugadores DEF y DEL. Por otra parte, también es necesario considerar la cantidad de jugadores FT3 en cada posición de juego lo cual podría influenciar las respuestas a las demandas físicas impuestas.

Respecto a la asociación entre las variables, la potencia muscular se estima indirectamente a partir de la altura del salto vertical (SJ y CMJ), estas variables se relacionaron significantemente con la distancia recorrida total (D), con la capacidad de realizar sprint en distancias cortas con cambio de dirección ( $\left.\mathrm{RSA}_{\mathrm{m}}\right)$ y sprint de forma repetida en corto intervalo de tiempo (RSA $)$. También se describió una relación con la agilidad (AG), indicando que la potencia muscular de miembros inferiores se presenta como un importante marcador de desempeño en acciones que involucran una alta intensidad. Tales resultados pueden estar asociados a que los atletas con PC con un menor compromiso motor, presentan mayor capacidad de tener elevados niveles de potencia anæeróbica, así también una menor influencia de factores neuromusculares propios del déficit motor (K loyiam et al., 2011; Yanci et al. , 2018). Corroborando con estos resultados, de Groot et al. (2012) identifica ron que los atletas con PC presentaron relaciones signi- ficativas entre lafuerza muscular y lacapacidad de sprint, sugiriendo que el rendimiento de la capacidad aeróbical anaeróbica en extremidades inferiores se encuentran más limitada por la reducción de la fuerza muscular en sí misma que por otros factores. Junto con esto, Reina et al. (2020), describió una relación positiva entre la capacidad de realizar cambios de dirección y la realiza ción de aceleraciones a una alta intensidad, así también, entre la capacidad de coordinación y la velocidad máxima al canzada en competencia por jugadores con distinto nivel de compromiso de acuerdo a su CD (Reina et al., 2020). Estudios realizados en atletas de fútbol sin discapacidad observaron asociaciones significativas entre los niveles de potencia muscular de tren inferior con la capacidad de realizar sprint en distancias cortas, concluyendo que el aumento de la fuerza máxima afecta el aumento de la potencia en extremidad inferior, pudiéndose asociar al aumento de la capacidad de aceleración, junto con la velocidad del desplazamiento con y sin cambios de dirección (W isløff et al., 2004).

También se observó que los participantes que presentaron una mayor distancia recorrida en la prueba de capacidad aeróbica, fueron significativamente más ágiles $(A G)$, veloces $\left(R_{S A}\right)$ y presentaron mejores tiempos promedios para realizar sprint repetidos $\left(\mathrm{RSA}_{\mathrm{p}}\right)$, indicando que el compromiso motor podría impactar y reducir en la capacidad de realizar acciones motoras de alta intensidad. Estas relaciones entre las variables físicas también se observa como un comportamiento similar a los atletas sin discapacidad. Strøyer, Hansen, \& Klausen (2004) identificaron la importancia del metabolismo aeróbico como un factor predominante en modalidades colectivas que requieren de un esfuerzo intermitente y que influencian significativamente la mantención de niveles elevados de intensidad durante el juego, esto para generar una recuperación más efectiva y rápida de los aspectos metabólicos frente a los estímulos intensos (C astagna, D'O ttavio, Vera, \& Álvarez, 2009; Tomlin \& Wenger, 2001). Sumado a eso, y en línea a nuestros resultados, Rampinini et al. (2007) reportan que la capacidad de realizar sprint repetido presenta relaciones significativas con la distancia total recorrida durante juegos oficiales. Para las asociaciones entre las variables de desempeño con la CD, se observaron relaciones significativas con las variables de $A G$, $R S A_{p^{\prime}}, R S A_{m}$ y la D, indicando que a mayor compromiso motor (menor CD) menos ágiles, veloces y resistentes son los atletas. Por otro lado, los indicadores de saltos verticales, sea con o sin la técnica de contra movimiento, no presentaron la misma tendencia ya que se obser- 
vó una amplia variación de resultados entre los grupos, así como en el número de participantes por CD lo cual puedehaber limitado resultados más contundentes. Propuestas para futuros estudios en el ámbito, podrían involucrar identificar los efectos de distintos tipos de entrenamiento en futbolistas con PC sobre las variables presentemente descritas. Las implicancias de estos resultados pueden ser de un gran uso para los entrenadores los cuales deben considerar las características específicas de cada CD en conjunto con la posición de juego y la táctica del equipo en sí misma, conjugando una serie de factores que busquen lograr los más altos niveles de rendimiento físico y deportivo.

\section{Referencias}

Ahmed, O. H., H ussain, A.W., Beasley, I., Dvorak, J., \&Weiler, R. (2015). Enhancing performance and sport injury prevention in disability sport: M oving forwards in the field of football. British Journal of Sports Medicine, 49(9), 566-567. doi.org/ 10.1136/ bjsports-2013-093058

Bangsbo, J. (2014). Physiological Demands of Football. SportsScience, 27(125), 1-6.

Bangsbo, J., laia, F. M. ., \& Krustrup, P. (2008).TheYo-Yo Intermittent RecoveryTest - Review Article. Sports M edicine, 38(1), 37-51.

Barrett, R. S. \& Lichtwark, G. A. (2010). Grossmusclemorphology and structure in spastic cerebral palsy: a systematic review. Developmental Medicine and Child Neurology, 52 (9), 794804. doi.org/ 10.1111/ j.1469-8749.2010.03686.x

Bishop, D. , Girard, 0., \& M endez-Villanueva, A. (2011). Repeatedsprint ability - part II: recommendations for training. Sports Medicine, 41(9), 741-756. doi.org/ 10.2165/ 11590560000000000-00000

Bosco, C., Luhtanen, P., \& Komi, P.V. (1983). A simplemethod for measurement of mechanical power in jumping. European Journal of Applied Physiology and Occupational Physiology, 50 (2), 273-282.doi.org/ 10.1007/ BF00422166

Boyd, C., Barnes, C., Eaves, S. J., M orse, C. I., Roach, N., \& W illiams, A. G. (2016). A time-motion analysis of Paralympic football for athletes with cerebral palsy. International Journal of SportsScienceand Coaching, 11(4), 552-558. doi.org/ 10.1177/ 1747954116654786

Buchheit, M., M endez-Villanueva, A., Delhomel, G., Brughelli, M ., \&Ahmaidi, S. (2010). Improving Repeated SprintA bility in Young Elite Soccer Players: Repeated Shuttle SprintsVs. Explosive StrengthTraining. Journal of Strength and Conditioning Research, 24(10), 2715-2722. doi.org/10.1519/ JSC.0b013e3181bf0223

Castagna, C., D'0 ttavio, S., Vera, J. G. \& \& Álvarez, J. C. B. (2009). Match demands of professional Futsal: $A$ case study. Journal of Scienceand M edicinein Sport, 12 (4), 490-494. doi. org/10.1016/ j.jsams. 2008.02.001

Castagna, C., Impellizzeri, F. M., Chamari, K., Carlomagno, D., \& Rampinini, E. (2006). Aerobic fitness and yo-yo continuous and intermittent tests performances in soccer players: A correlation study. Journal of Strength and Conditioning Research,
20(2), 320-325. doi.org/ 10.1519/ R-18065.1

Chaouachi, A., M anzi,V., Chaalali, A., Wong, D. P., Chamari, K., \& Castagna, C. (2012). Determinants analysis of change-ofdirection ability in elite soccer players. Journal of Strength and Conditioning Research, 26(10), 2667-2676. doi.org/ 10.1519/ JSC. 0b013e318242f97a

Cohen, J. (1988). Statistical power analysis for the behavioral sciences. Hillsdale, N. J.: L. ErlbaumAssociates.

Gamonales, J. M., León, K., Jiménez, A., \& Muñoz-Jiménez, J. (2019). Indicadores de rendimiento deportivo en el fútbol-7 para personas con parálisis cerebral / Sport Performance Indicators in Football 7-A-Sidefor Peoplewith Cerebral Palsy. Revista Internacional de M edicina y Ciencias de La Actividad Fisica y Del Deporte, 19(74), 309-328.doi.org/ 10.15366/ rimcafd2019.74.009

Girard, O., Mendez-Villanueva, A., \& Bishop, D. (2011). Repeatedsprint ability - part 1: factors contributing to fatigue. Sports Medicine, 41(8), 673-694. doi.org/ 10.2165/ 11590550000000000-00000

Gorla, J. I., Nogueira, C. D., Gonçalves, H. R., De Faria, F. R., Buratti, J. R., Nunes, N., Pereira do Rêgo, J. T., Borges, M., Vieira, I. B., \& Labrador Roca, V. (2018). Composición corporal y perfil somatotípico dejugadoresbrasileños de fútbol siete con ParálisisCerebral de acuerdo con laclasificación funcional. Contribución al DeporteParalímpico (Body composition and somatotype profile of football-seven Brazilian p. Retos, (35), 326-328. doi.org/ 10.47197/ retos. v0i35.58931

Graham, H. K., Rosenbaum, P., Paneth, N., Dan, B., Lin, J.-P., Damiano, Di. L., Becher, J. G., Gaebler-Spira, D., Colver, A., Reddihough, D. S., Crompton, K. E., Lieber, R. L. (2016). Cerebral pal sy. N ature ReviewsD iseasePrimers, 2, 15082. doi.org/ 10.1038/ nrdp.2015.82

deGroot, S., Dallmeijer, A. J., Bessems, P. J., Lamberts, M. L., van der Woude, L. H., \& Janssen, T. W. (2012). Comparison of muscle strength, sprint power and aerobic capacity in adults with and without cerebral palsy. Journal of rehabilitation medicine, 44(11), 932- 938. https: / / doi.org/ 10.2340/ 165019771037

Horicka, P., Hianik, J., \& Šimonek, J. (2014). The relationship between speed factors and agility in sport games. Journal of Human Sport and Exercise, 9(1), 49-58. doi.org/ 10.4100/ jhse. 2014.91.06

Hussain, A.W., O nambele, G. L., W illiams, A. G., \& M orse, C. I. (2014). M usclesize, activation, and coactivation in adultswith cerebral palsy. M uscle\& N erve, 49 (1), 76-83. doi.org/ 10.1002/ mus. 23866

IFCPF, International Federation of Cerebral Palsy Football (2018a). Modifications to the laws of the game. International Federation of CP Football, 1-8.

IFCPF. (2018b). Classification Rules and Regulations. International Federation of CP Football, 1-113.

Kloyiam, S., Breen, S., Jakeman, P., Conway, J., \& Hutzler, Y. (2011). Soccer-specific endurance and running economy in soccer players with cerebral palsy. Adapted Physical Activity Quarterlyl :APAQ, 28(4), 354-367.

Köklü,Y., Asçi, A., Koçak, F. Ü., Alemdarođlu, U., \& Dündar, U. (2011). Comparison of the Physiological Responsesto Different Small-Sided Games in EliteYoung Soccer Players. Journal of 
Strength and Conditioning Research, 25(6), 1522-1528. doi. org/ 10.1519/JSC. 0b013e3181e06ee1

Lieber, R. L., \& Fridén, J. (2019). M uscle contracture and passive mechanicsin cerebral palsy. Journal of Applied Physiology, 126 (5), 1492-1501. doi.org/ 10.1152/ japplphysiol.00278.2018

Loturco, I., D¹/4Angelo, R. A., Fernandes,V., Gil, S., Kobal, R. , Cal Abad, C. C.,... Nakamura, F.Y. (2015). Relationship Between Sprint Ability and Loaded/ Unloaded Jump Tests in Elite Sprinters. Journal of Strength and Conditioning Research, 29(3), 758- 764. doi.org/ 10.1519/ JSC.0000000000000660

M ohr, M. , Krustrup, P., \& Bangsbo, J. (2003). Match performance of high-standard soccer players with special reference to development of fatigue. Journal of Sports Sciences, 21(7), 519528. doi.org/ 10.1080/ 0264041031000071182

Moreira, P. E. D., Sousa, R. B. e, Morales, J. C. P., Greco, P. J., Arroyo, M. P. M., \& Praça, G. M. (2021). Comportamiento táctico de jugadores de fútbol de diferentes posiciones, durante unatemporada deportiva. Retos, 2041, 1-6.

Morgans, R., Orme, P.,Anderson, L., \& Drust, B. (2014). Principles and practices of training for soccer. Journal of Sport and $\mathrm{H}$ ealth Science, 3(4), 251-257. doi.org/ 10.1016/ j.jshs. 2014.07.002

Mujika, I., Santisteban, J., Impellizzeri, F. M., \& Castagna, C. (2009). Fitness determinants of successin men's and women's football. Journal of Sports Sciences, 27(2), 107-114.doi.org/ 10.1080/02640410802428071

Pastor, D., Campayo-Piernas, M. , Pastor, J. T., \& Reina, R. (2019). A mathematical model for decision-making in the classification of para-footballers with different severity of coordination impairments. Journal of Sports Sciences, 37(12), 1403-1410. doi.org/ 10.1080/ 02640414.2018.1560617

Rampinini, E., Bishop, D., Marcora, S. M., Ferrari Bravo, D., Sassi, R., \& Impellizzeri, F. M. (2007).Validity of simple field tests as indicators of match-related physical performance in top-level professional soccer players. International Journal of Sports $\mathrm{Me}$ dicine, 28(3), 228-235.doi. org/ 10.1055/ s-2006-924340

Reina, R., Iturricastillo, A., Castillo, D., U rbán, T. , \&Yanci, J. (2020). Activity limitation and match load in para-footballers with cerebral palsy: An approach for evidence-based classification. Scandinavian Journal of Medicine and Science in Sports, 30(3), 496-504. doi.org/ 10.1111/ sms. 13583

Reina, R., Iturricastillo, A., Sabido, R., Campayo-Piernas, M., \& Yanci, J. (2018). Vertical and Horizontal Jump Capacity in International Cerebral Palsy Football Players. International Journal of Sports Physiology and Performance, 13(5), 597603. doi.org/ 10.1123/ ijspp. 2017-0321

Reina, R., Sarabia, J. M., Caballero, C., \&Yanci, J. (2017). How does the ball influence the performance of change of direction and sprint tests in para-footballers with brain impairments? Implications for evidence-based classification in CP-Football. PLOS ONE, 12(11), e0187237.doi.org/10.1371/ journal. pone. 0187237

Reina, R., Sarabia, J. M., Yanci, J., GarcíaVaquero, M. P., \& Campayo-Piernas, M. (2016). Change of Direction Ability Performance in Cerebral Palsy Football Players According to Functional Profiles. Frontiers in Physiology, 6, 1-8. doi.org/ 10.3389/ fphys. 2015.00409

Rhea, M. R. (2004). D etermining the Magnitude of Treatment Effects in StrengthTraining ResearchThrough the U se of the Effect Size. The Journal of Strength and Conditioning Research,
18(4), 918.doi.org/ 10.1519/14403.1

Runciman, P., Tucker, R., Ferreira, S., Albertus-Kajee, Y., \& Derman, W. (2016). Effects of Induced Volitional Fatigue on Sprint and Jump Performance in Paralympic Athletes with Cerebral Palsy. American Journal of Physical Medicine \& Rehabilitation, 95(4), 277-290. doi.org/ 10.1097/ PHM.0000000000000372

Sporis, G. , Jukic, I., O stojic, S. M., \& Milanovic, D. (2009). Fitness Profiling in Soccer: Physical and Physiologic Characteristics of Elite Players. Journal of Strength and Conditioning Research, 23 (7), 1947-1953. doi.org/ 10.1519/ JSC.0b013e3181b3e141

Stølen, T., Chamari, K., Castagna, C., \& W isløff, U. (2005). Physiology of soccer: An update. Sports Medicine, 35 (6), 501536. doi.org/ 10.2165/ 00007256-200535060-00004

Strøyer, J., Hansen, L., \& K lausen, K. (2004). Physiological Profile and Activity Pattern of Young Soccer Players during $M$ atch Play. M edicine \& Science in Sports \& Exercise, 36(1). Retrieved from https:/ / journals. Iww. com/ acsm-msse/ Fulltext/ 2004/ 01000Physiological_Profile_and_Activity_Pattern_of.28. aspx Tomlin, D. L., \&Wenger, H. A. (2001). The Relationship Between Aerobic Fitness and Recovery from High Intensity Intermittent Exercise. Sports Medicine, 31(1), 1-11. doi.org/ 10.2165/ 00007256-200131010-00001

Tweedy, S. M., \& Vanlandewijck, Y. C. (2011). International Paralympic Committee position stand-background and scientific principles of classification in Paralympic sport. British Journal of Sports M edicine, 45 (4), 259-269. doi.org/ 10.1136/ bjsm. 2009.065060

Verschuren, O., Smorenburg, A. R. P., Luiking,Y., Bell, K., Barber, L., \& Peterson, M. D. (2018). Determinants of muscle preservation in individuals with cerebral palsy across the lifespan: anarrative review of the literature. Journal of Cachexia, Sarcopenia and Muscle, 9(3), 453-464.doi.org/ 10.1002/ jcsm. 12287

Willick, S. E., Webborn, N., Emery, C., Blauwet, C. A., PitGrosheide, P., Stomphorst, J., ... Schwellnus, M. (2013). The epidemiology of injuries at the London 2012 Paralympic Games. British Journal of Sports Medicine, 47(7), 426-432. doi.org/ 10.1136/ bjsports-2013-092374

Wisløff, U., Castagna, C., Helgerud, J., Jones, R., \& Hoff, J. (2004). Strong correlation of maximal squat strength with sprint performance and vertical jump height in elite soccer players. British Journal of Sports M edicine, 38(3), 285-288. doi.org/ 10.1136/ bjsm. 2002.002071

Yanci, J., Castagna, C., Los Arcos, A., Santalla, A., Grande, I., Figueroa, J., \& Camara, J. (2016). M uscle strength and anaerobic performance in football players with cerebral palsy. Disability and H ealth Journal, 9 (2), 313-319. doi.org/ 10.1016/ j.dhjo.2015.11.003

Yanci, J., Castillo, D., Iturricastillo, A., \& Reina, R. (2019). Evaluation of the $O$ fficial M atch External Load in Soccer PlayersW ith Cerebral Palsy. Journal of Strength and Conditioning Research, 33(3), 866-873. doi.org/ 10.1519/ JSC.0000000000002085 Yanci, J., Castillo, D., Iturricastillo, A., U rbán, T., \& Reina, R. (2018). External M atch Loads of FootballersW ith Cerebral Palsy: A ComparisonAmong Sport Classes. International Journal of Sports Physiology and Performance, 13(5), 590-596. doi.org/ 10.1123/ ijspp. 2017-0042 